\title{
Transition from Interictal to Ictal Activity in Limbic Networks In Vitro
}

\author{
Volodymyr I. Dzhala and Kevin J. Staley \\ Department of Neurology and Pediatrics, University of Colorado Health Sciences Center, Denver, Colorado 80262
}

The transition from brief bursts of synchronous population activity characteristic of interictal epileptiform discharges (IEDs) to more prolonged epochs of population activity characteristic of seizures (ictal-like activity) was recorded in juvenile rat hippocampal- entorhinal cortex slices and hippocampal slices using multiple-site extracellular electrodes. Epileptiform activity was elicited by either increased extracellular potassium or 4-AP. IEDs originated in the CA3 a- $\mathrm{b}$ region and spread bidirectionally into CA1 and CA3c dentate gyrus. The transition from IEDs to ictal-like sustained epileptiform activity was reliably preceded by (1) increase in IED propagation velocity, (2) increase in IED secondary afterdischarges and their reverberation between CA3a and CA3c, and (3) shift in the IED initiation area from CA3 $\mathrm{a}-\mathrm{b}$ to CA3c. Ictal-like sustained network oscillations $(10-20 \mathrm{~Hz})$ originated in CA3c and spread to CA1. The pattern of hippocampal ictal-like activity was unaffected by removal of the entorhinal cortex. These findings indicate that interictal and ictal activity can originate in the same neural network, and that the transition from interictal to ictal-like-sustained activity is preceded by predictable alterations in the origin and spread of IEDs. These findings elucidate new targets for investigating the proximate causes, prediction, and treatment of seizures.

Key words: epileptiform activity; entorhinal cortex; hippocampus; CA3; CA1; dentate gyrus

\section{Introduction}

Interictal epileptiform discharges (IEDs) in the human electroencephalogram (EEG) are an important diagnostic feature of temporal lobe epilepsy (Pedley, 1980), although they are of little use for short-term seizure prediction (Gotman, 1991). Seizures are preceded by increases in the extracellular potassium concentration (Pedley et al., 1976), and in vitro studies have confirmed a corresponding pre-ictal depolarization of the neuronal membrane potential (Jensen and Yaari, 1997). More recently, nonlinear time series analyses of EEG activity have demonstrated changes in brain wave activity minutes before a seizure (Martinerie et al., 1998; Lehnertz et al., 2001). Together, these observations suggest that the operations of epileptic neural networks undergo predictable alterations before a seizure (Prince et al., 1983). However, there are no clinically accessible and physiologically interpretable parameters that can be used to address a fundamental question: What changes in the epileptic network lead to seizures? Complicating this question is a paucity of direct evidence that IEDs and seizures arise from the same neural network (Barbarosie and Avoli, 1997; Bragin et al., 1997).

We addressed these questions in the hippocampal-entorhinal brain slice preparation, in which the cellular and network activities corresponding to ictal and interictal discharges have been extensively characterized (Schwartzkroin and Prince, 1977). The

Received March 6, 2003; revised June 26, 2003; accepted July 1, 2003.

This work was supported by a grant from the National Institutes of Health-National Institute of Neurological Disorders and Stroke.

Correspondence should be addressed to Dr. Kevin J. Staley, Department of Neurology and Pediatrics, University of Colorado Health Sciences Center, 4200 East Ninth Avenue, B182, Denver, C0 80262. E-mail: Kevin.Staley@UCHSC.edu. Copyright $\odot 2003$ Society for Neuroscience $\quad 0270-6474 / 03 / 237873-08 \$ 15.00 / 0$ entorhinal cortex-hippocampal formation is involved in the generation of seizures that originate in the temporal lobes (Lothman, 1994; McNamara, 1994). Despite numerous studies on seizure circuits in the entorhinal cortex and hippocampus (Walther et al., 1986; Jones and Heinemann, 1988), the roles of these two structures in seizures initiation is incompletely understood (Heinemann et al., 1993; Jones, 1993). In some in vitro models, the ictal-like epileptiform discharges originate in the entorhinal cortex with limited involvement of the hippocampus (Dreier and Heinemann, 1990; Weissinger et al., 2000), or may propagate via the dentate gyrus to the hippocampus proper from where they re-enter the entorhinal cortex (Rafiq et al., 1993; Barbarosie et al., 2000). In other conditions, the CA 3 region acts as the pacemaker, whereas ictal activity is limited to CA1 (Jensen and Yaari, 1988; Traynelis and Dingledine, 1988).

In vivo animal models and findings from human epilepsy surgery indicate that chronic epilepsy is associated with hippocampal cell loss and synaptic reorganization (Liu et al., 1994; Buckmaster and Dudek, 1997; Holmes and Ben Ari, 1998) and that resection eliminates or reduces the frequency of seizures in most patients with medically intractable partial complex seizures (Ojemann, 1987; Wieser et al., 1993). However, some temporal lobe epilepsies involve interactions between entorhinal and hippocampal regions (Spencer and Spencer, 1994), and many patients have extrahippocampal seizures (Bautista et al., 1999).

In vivo animal models suggest that seizures originate predominantly in the hippocampus (Bragin et al., 1999b). Epileptic afterdischarges can arise in the CA3-CA1 circuits in vivo, and reverberation through the hippocampal-entorhinal loop is not critical (Bragin et al., 1997). The goal of the present investigation was to reveal the relationship between ictal and interictal activity in hip- 
pocampal regions (CA1, CA3a, CA3b, CA3c) when these activities were induced by increased extracellular potassium concentration in the hippocampal-entorhinal and hippocampal slices in vitro.

\section{Materials and Methods}

Experimental systems. Hippocampal-entorhinal cortex slices were prepared from male Wistar rats at postnatal day 10 (P10) to P20. The first 24 hr after birth were designated P0. All animal use protocols conformed to the University of Colorado Health Sciences Center animal care use committee and the National Institutes of Health guidelines on the use of laboratory animals. Animals were anesthetized with chloral hydrate (350 $\mathrm{mg} / \mathrm{kg}$, i.p.) and decapitated. The brain was rapidly removed and placed in an oxygenated $\left(95 \% \mathrm{O}_{2} / 5 \% \mathrm{CO}_{2}\right)$, ice-cold artificial CSF ( $\mathrm{aCSF}$ ) of the following composition (in $\mathrm{mm}$ ): $126 \mathrm{NaCl}, 3.5 \mathrm{KCl}, 2 \mathrm{CaCl}_{2}, 1.3 \mathrm{MgCl}_{2}$, $25 \mathrm{NaHCO}_{3}, 1.2 \mathrm{NaH}_{2} \mathrm{PO}_{4}$, and 11 glucose at a $\mathrm{pH}$ of 7.4. Hippocampalentorhinal cortex transverse slices (thickness, $0.5-0.6 \mathrm{~mm}$ ) were cut with a vibroslicer (Leica VT 1000S; Leica Microsystems, Nussloch, Germany) and kept in oxygenated $\left(95 \% \mathrm{O}_{2} / 5 \% \mathrm{CO}_{2}\right)$ aCSF at room temperature $\left(20-22^{\circ} \mathrm{C}\right)$ at least $1 \mathrm{hr}$ before use.

Electrophysiological recordings and data analysis. For electrophysiological recordings, individual slices were transferred to a conventional submerged chamber and superfused continuously with oxygenated aCSF at $32^{\circ} \mathrm{C}$ at a rate of $2-3 \mathrm{ml} / \mathrm{min}$. Hippocampal-entorhinal cortex transverse slices posterior to the midtemporal (caudal) part of the hippocampus (plates, 100-109; according to Paxinos and Watson, 1986) were used in the study. Extracellular field potential recordings were performed in the pyramidal cell layer of the CA3a, CA3b, CA3c, and CA1 subfields and the granular cell layer of the dentate gyrus. A series of cutting experiments were performed using a micro-knife to establish the contribution of various regions to generation and maintenance of epileptiform activity.

Extracellular field potentials were recorded using tungsten microelectrodes and multichannel amplifier $(1000 \times)$ with enhanced electromagnetic interference noise suppression developed for neurobiology research applications. Using microelectrodes made from coated tungsten wire of $50 \mu \mathrm{m}$ diameter (California Fine Wire Company, Grover Beach, CA) enables simultaneous recordings of multiple unit activity (MUA; $500 \mathrm{~Hz}$ high-pass filter) and population field activity (EEG band, 1-100 $\mathrm{Hz}$ ) from 10 to hundreds of neurons nearby each electrode (Cohen and Miles, 2000). Root mean square (RMS) noise level with an electrode placed in the perfusion solution was typically $4-5 \mu \mathrm{V}$, whereas the amplitude of action potentials recorded from the stratum pyramidale ranged from this noise level up to $200 \mu \mathrm{V}$. The signals were digitized using an analog-to-digital converter (DigiData 1322A; Axon Instruments, Foster City, CA). Sampling interval per signal was $100 \mu \mathrm{sec}(10$ $\mathrm{kHz}$ ). Signals were filtered with a $0.1 \mathrm{~Hz}$ to $4 \mathrm{kHz}$ bandpass; wide-band recordings in the results refer to this frequency range. Axoscope (Axon Instruments), Mini Analysis Program (Synaptosoft, Decater, GA), and Origin (OriginLab, Northampton, MA) programs were used for the acquisition and data analysis. The MUA was determined from $500 \mathrm{~Hz}$ high-pass-filtered (single pole, RC filter) raw data with a spike detection algorithm (Mini Analysis Program) and verified visually. Spikes with amplitude greater than three times the RMS noise level were accepted. Population field activity was determined from 1 to $100 \mathrm{~Hz}$ band-passfiltered (eight-pole, Bessel filter) raw data. The onset of synchronous population activity was timed from the negative peak of the initial population spike recorded in the principal cell layer (Fig. 2). Crosscorrelation between population activity in different hippocampal regions was performed using the timing of the population spikes.

Power spectrum analysis, revealing the power levels of different frequency components in the signal, was performed after applying a Hamming window function. Power was calculated in each 20 sec time window preceding transition from interictal to ictal-like activity by integrating the RMS value of the signal in frequency bands from 1 to $100 \mathrm{~Hz}$ (EEG band) and from 200 to $500 \mathrm{~Hz}$ (fast field ripple band). Records with $200-300 \mathrm{sec}$ interictal phase duration were selected for statistical power analysis.

Statistical analysis. Group measures are expressed as mean \pm SEM;
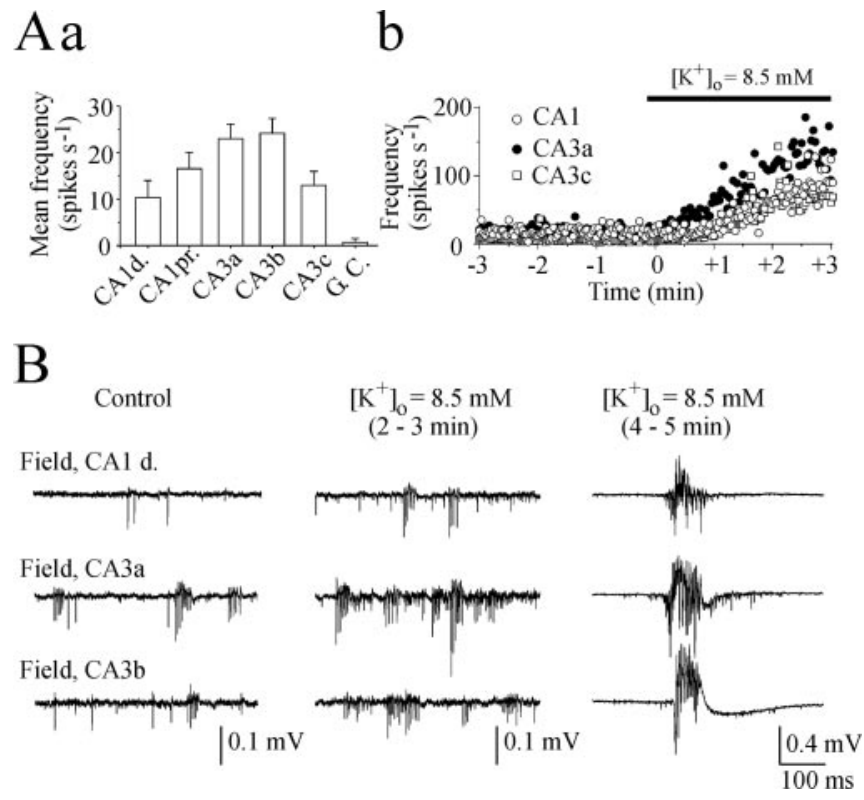

Figure 1. Effects of $8.5 \mathrm{~mm}\left[\mathrm{~K}^{+}\right]_{0}$ on spontaneous activity of CA3 and CA1 cells. Aa, Averaged frequency of spontaneous MUA in the pyramidal cell layer of different CA3 and CA1 areas and in the granular cell layer of the dentate gyrus in a P15-P16 hippocampal-entorhinal cortex slices. $A b$, Progressive increase in spike frequency as $\left[\mathrm{K}^{+}\right]_{0}$ was increased to $8.5 \mathrm{~mm}$. $B$, Bath application of $8.5 \mathrm{~mm}\left[\mathrm{~K}^{+}\right]_{0}$ increased neuronal firing rate, resulting in synchronous network-driven population bursts. Selected traces depict extracellular activity in the CA3a, CA3b, and distal CA1 pyramidal cell layer in control and after high $\mathrm{K}^{+}$application. Separation distance between electrodes, $-600 \mu \mathrm{m}$; recording range, $0.1 \mathrm{~Hz}$ to $4 \mathrm{kHz}$.

error bars also indicate SEM. The statistical significance of differences was assessed with the Student's $t$ test. The level of significance was set at $p<0.05$.

Drugs. Drugs were purchased from Sigma-RBI (St. Louis, MO) and Tocris (Ellisville, MO), prepared as stock solutions, and stored aliquoted in tightly sealed vials at recommended temperatures. During the experiments, thawed aliquots were kept on ice and protected from light until use.

\section{Results}

Evolution of the ictal epileptiform activity in the hippocampal-entorhinal cortex slices

We performed multisite extracellular field potential recordings in the combined hippocampal-entorhinal cortex slice preparation in vitro. Spontaneous unit activity was always evident in simultaneous records from the pyramidal cell layer of the CA3a, CA3b, CA3c, and CA1 hippocampal regions (Lorente de No, 1934). Spike amplitudes ranged from the noise level up to $10-200 \mu \mathrm{V}$, suggesting that electrodes detected activity from multiple neurons. The mean frequency of detected action potentials varied from $10.4 \pm 3.6 \mathrm{spikes} / \mathrm{sec}^{-1}$ at the CA1 area to $23 \pm 3.1$ spikes/ $\mathrm{sec}^{-1}$ at CA3a and $24.1 \pm 3.2 \mathrm{spikes} / \mathrm{sec}^{-1}$ at CA3b (Fig. $1 \mathrm{Aa}$; $n=8$ slices). In simultaneous records from the granular cell layer of the dentate gyrus, there was typically no spontaneous activity, although vertical movement of the recording electrode provoked transient firing. This suggests that the highest neuronal excitability is in the hippocampal CA3 network, as suggested by the extensive positive feedback mediated by recurrent axon collaterals of the pyramidal cells (MacVicar and Dudek, 1980; Miles and Wong, 1983).

Increasing the extracellular concentration of $\mathrm{K}^{+}$induced increasing discharge frequency of spontaneously active cells as well as recruitment of previously inactive cells to generate the action potentials (Cohen and Miles, 2000). Bath application of $8.5 \mathrm{~mm}$ $\mathrm{K}^{+}$(Traynelis and Dingledine, 1988) induced a progressive in- 

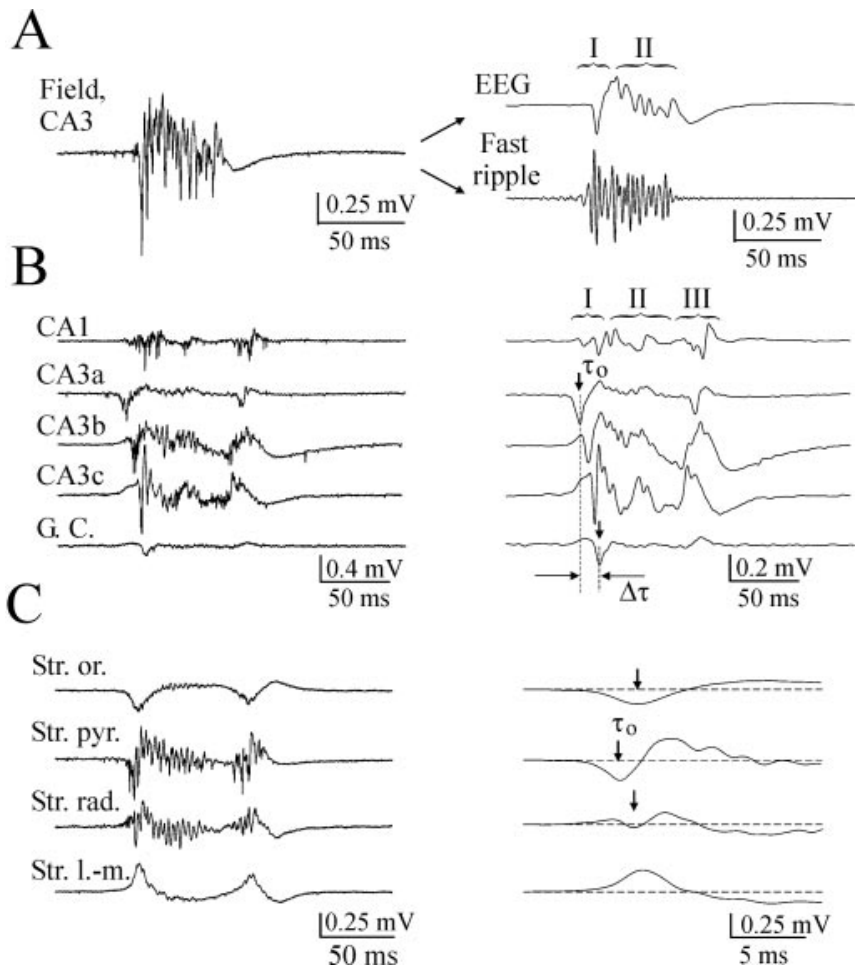

Figure 2. Quantification IEDs related extracellular potentials and analysis of IED origin and propagation. $A$, Example of interictal epileptiform discharge in hippocampal- entorhinal cortex slice of a P19 rat. Extracellular field potential recording (wide band, $0.1 \mathrm{~Hz}$ to $4 \mathrm{kHz}$ ) at the $\mathrm{CA} 3$ pyramidal cell layer before filtering (left trace) and after filtering (right traces) for EEG components of IED (1-100 Hz bandpass filter) and IED-related fast field ripple ( $200-500 \mathrm{~Hz}$ bandpass filter). EEG components are labeled (I, primary population spike; II, slow spike wave). $B$, Simultaneous extracellular field potential records in the pyramidal cell layer of the $\mathrm{CA3a}$, $C A 3 \mathrm{~b}, \mathrm{CA} 3 \mathrm{C}$, CA1, and granular cell layer (G. C.) of the dentate gyrus regions. Examples of IEDs before (left traces) and after (right traces) filtering in EEG band (raw data, $0.1 \mathrm{~Hz}$ to $4 \mathrm{kHz}$; EEG, 1-100 Hz). EEG components are labeled (I, primary population spike; II, slow spike wave; III, secondary afterdischarges). Shift of the negative peaks of primary (initial) population spike $(\Delta \tau)$ shows that the population spike is initiated in CA3a and bidirectionally propagates to CA3c dentate gyrus and CA1. C, Simultaneous extracellular records in the CA3a stratum oriens, stratum pyramidale, stratum radiatum, and stratum lacunosum-moleculare layers (wide band range, $0.1 \mathrm{~Hz}$ to $4 \mathrm{kHz})$. Filtered traces $(1-100 \mathrm{~Hz})$ show that the population spike is initiated in the somatic layer and propagated into dendritic trees and axons. $A-C$, IEDs induced by $8.5 \mathrm{~mm}\left[\mathrm{~K}^{+}\right]_{0}$.

crease of neuronal discharges in all recording areas and their synchronization, resulting in high-amplitude network-driven population bursts (Fig. $1 A, B ; n=12$ of 12 slices).

These population bursts had the typical features of IEDs (Pedley, 1980): they were abrupt in onset, primarily monophasic, and their duration was $<200 \mathrm{msec}$. Digital filtering and power spectra analysis revealed MUA (500-1000 Hz; data not shown) and fast field ripples $(200-500 \mathrm{~Hz})$, phase locked to locally derived field potential oscillation in EEG band (1-100 Hz) (Fig. 2A). EEG components of the typical IEDs consisted of primary (initial) population spike and slow spike wave (Fig. 2). Continuous application of high $\mathrm{K}^{+}$resulted in IEDs, followed by secondary afterdischarges. Secondary afterdischarges, lasting hundreds of milliseconds and composed of large-amplitude population spikes, were usually observed before transition to prolonged ictal-like activity. Similar field potentials also have been demonstrated in chronic seizure models in vivo (Bragin et al., 1999b) and in the epileptogenic region of patients with temporal lobe epilepsy (Bragin et al., 1999a).

Within 2-20 min, IEDs developed into ictal-like epileptiform activity (75\%; $n=9$ of 12 slices; Fig. 3A). Ictal-like epileptiform
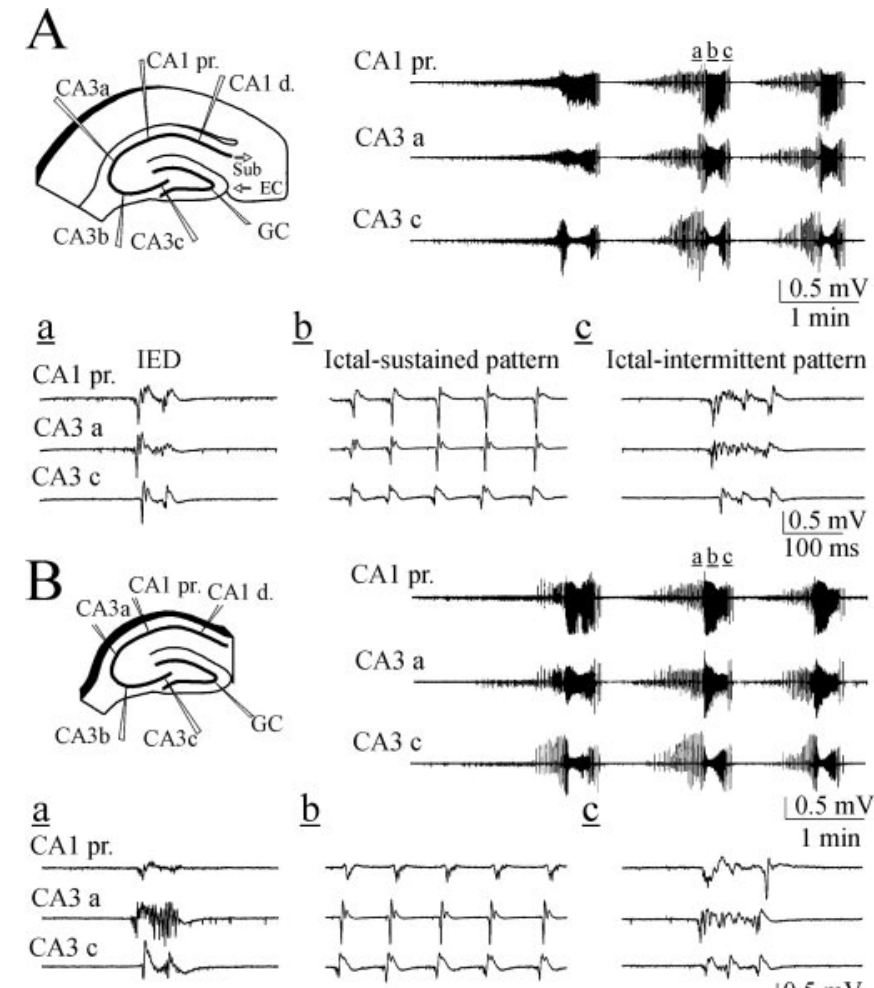

$\underline{b}$

$1 \mathrm{~min}$
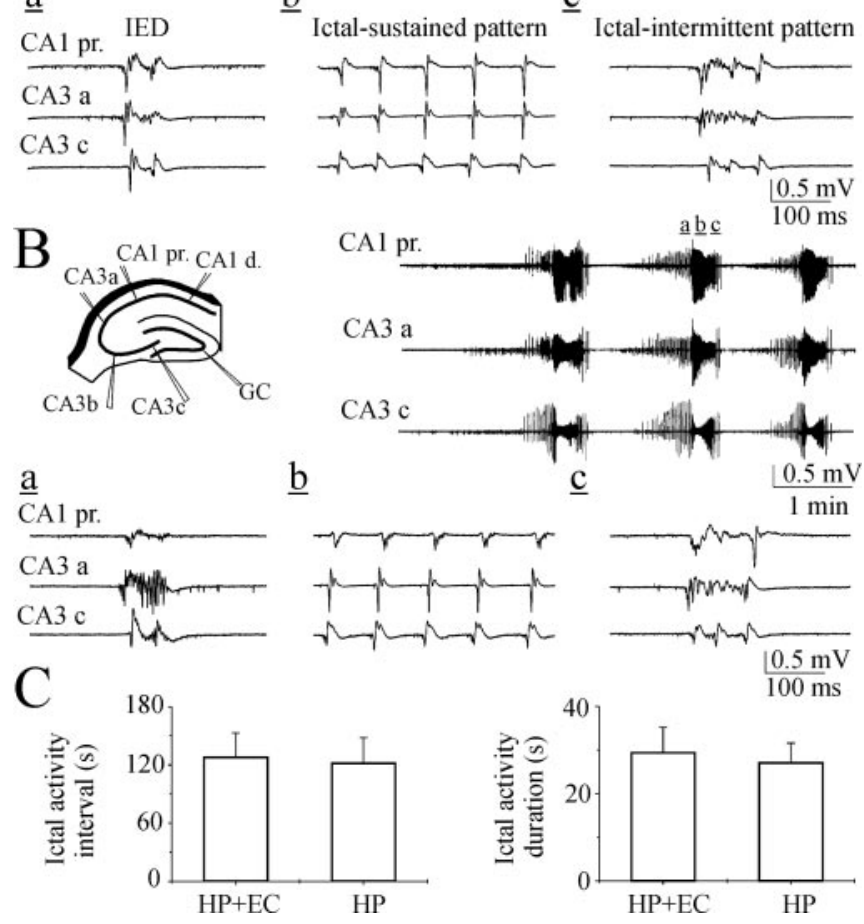

$\mathrm{CA} 3 \mathrm{c}$
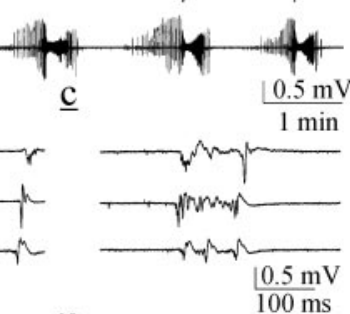

Figure 3. Hippocampal network is self-sufficient to generate recurrent ictal-like epileptiform activity. $A$, Scheme of extracellular field potential recordings (wide band, $0.1 \mathrm{~Hz}$ to $4 \mathrm{kHz}$ ) in the CA3a, CA3b, CA3c, and CA1 pyramidal cell layer in the hippocampal-entorhinal cortex slice of a P15 rat. Selected traces represent recurrent IEDs and ictal-like epileptiform discharges induced by continuous application of $8.5 \mathrm{~mm}\left[\mathrm{~K}^{+}\right]_{0}$. The events marked by small letters $(a-c)$ are shown on an extended time scale. $B$, Scheme of extracellular recordings from the same slice after removal of entorhinal cortex. Removing the entorhinal cortex does not affect $8.5 \mathrm{~mm}$ $\left[\mathrm{K}^{+}\right]_{0}$-induced recurrent ictal-like epileptiform activity. C, Averaged ictal activity duration and interval in the hippocampal slices before and after removal of entorhinal cortex. At the $p=0.05$ level, the two means are not significantly different.

activity consisted of ictal intermittent (clonic) patterns or initial ictal-sustained (tonic) and subsequently ictal intermittent (clonic) patterns of population discharges (Dzhala and Staley, 2003). The ictal-sustained pattern lasted $2-15 \mathrm{sec}$ and was characterized by rhythmic oscillations of $8-20 \mathrm{~Hz}$, gradually decreasing in frequency and increasing in duration. This pattern was followed by a series of intermittent discharges consisting of initial population spikes and a series of secondary afterdischarges that lasted for hundreds of milliseconds. The ictal discharges were followed by postictal depression with reduced MUA. Continuous application of $8.5 \mathrm{mM} \mathrm{K}^{+}$was associated with recurrent interictal- and ictallike epileptiform patterns at regular intervals of 2-5 min within a given slice.

The interictal and ictal-like epileptiform patterns induced by high potassium were aggravated by elevating the temperature. Increasing the aCSF temperature from $32^{\circ}$ to $37^{\circ} \mathrm{C}$ decreased the inter-IED interval by $17.9 \pm 2.4 \%(p=0.04)$ and the interval between ictal-like activities by $13.4 \pm 3.3 \%(n=3 ; p=0.023)$. 


\section{Hippocampal network is self-sufficient to generate recurrent ictal-like activity}

After the initial recordings in the hippocampal-entorhinal cortex slices, the entorhinal cortex was removed, dissecting the perforant path inputs to all regions of the hippocampus and dentate gyrus. We next performed multisite extracellular field potential recordings in the isolated hippocampal slice preparations (Fig. $3 B)$. Removal of the entorhinal cortex from the hippocampalentorhinal cortex slices did not affect recurrent ictal-like epileptiform patterns in the hippocampal slices. All recurrent ictal-like episodes consisted of an ictal intermittent pattern or initial ictalsustained and subsequently ictal-intermittent patterns recorded synchronously in the CA3-CA1 and dentate gyrus circuitries and followed by postictal depression. In the hippocampal-entorhinal cortex slices from P12-P16 rats, mean ictal-like activity duration and interval were $29.4 \pm 5.8 \mathrm{sec}$ and $128 \pm 25 \mathrm{sec}(n=9$ slices $)$. After removal of the entorhinal cortex, mean ictal-like activity duration and interval in the cut hippocampal slices were similar, $27.1 \pm 4.58 \sec (n=9 ; p=0.759)$ and $121.9 \pm 26 \sec (n=9 ; p=$ 0.86 ), correspondingly (Fig. $3 C$ ). These findings suggest that: (1) ictal-like epileptiform activity independently arises in the hippocampal circuitries; and (2) reverberation of excitation in the hippocampal-entorhinal cortex loop is not critical for the maintenance of recurrent ictal-like patterns.

\section{Pre-ictal reorganization of hippocampal network activity}

In agreement with previous studies in neonatal (Luhmann et al., 2000) and adult (Korn et al., 1987; Colom and Saggau, 1994; Jensen and Yaari, 1997) rats, IEDs originate in the CA3 region and spread from there into CA1. Comparison of the time of negative peak of the initial population spikes in the pyramidal cell layer of the CA3a, CA3b, CA3c, proximal, and distal CA1 subregions and the granular cell layer of the dentate gyrus in the hippocampal slice preparations demonstrated that $95 \%$ of the IEDs were initiated in the $\mathrm{CA} 3 \mathrm{a}-\mathrm{CA} 3 \mathrm{~b}$ subregion and bidirectionally propagated to the CA1 and CA3c-hilus areas (Figs. 2, 5). Four to $5 \%$ of the IEDs were initiated in the CA3b-CA3c subfields, and $<1 \%$ of the IEDs were initiated at the proximal CA1 area. Measuring the distances between electrodes and averaged time delays between initial population spikes, the mean speed of IED propagation in the interictal state was $40.8 \pm 8 \mathrm{~mm} / \mathrm{sec}(n=8)$.

In agreement with previous studies on stimulus-induced epileptiform discharges in vivo (Bragin et al., 1997), power spectra analysis reveals several distinctive epochs of the population network activity: (1) 2-40 Hz oscillations in the EEG band; (2) $80-$ $120 \mathrm{~Hz}$ band oscillations; and (3) 200-400 Hz oscillations representing fast field ripples (Fig. 4). Spectral analysis of the interictal epileptiform activity in each $20 \mathrm{sec}$ window preceding transition to ictal-like activity reveals a slow gradual increase of IED power in the EEG band and in the fast field ripple band $(200-400 \mathrm{~Hz})$ (Fig. 4). However, the variability in this spectral shift was too large in relation to the size of the shift to be a reliable indicator of the pre-ictal state (Fig. 4C). Interictal versus pre-ictal states were also not distinguishable by IED intervals $(1.56 \pm 0.11 \mathrm{sec}$ vs $1.63 \pm 0.12 \mathrm{sec}$ for the $60 \mathrm{sec}$ period before ictal-like activity onset; $n=8, p=0.7$ ).

A remarkable feature of the pre-ictal phase was a gradual decrease of the propagation time of population spikes from the $\mathrm{CA} 3 \mathrm{a}$ to CA3c areas from $28.7 \pm 5.1 \mathrm{msec}$ to $12.2 \pm 1.4 \mathrm{msec}(n=$ $7 ; p=0.008$, variance, 179 and 13 , correspondingly). The velocity of propagation increased to $91 \pm 7.8 \mathrm{~mm} / \mathrm{sec}(n=7)$. In the 60 sec preceding ictal-like sustained discharges, $37.6 \pm 4.4 \%(n=7)$ of primary population bursts were initiated in the CA3c-hilar region and propagated to CA3a and CA1 (Figs. 5, 6). IED dura-

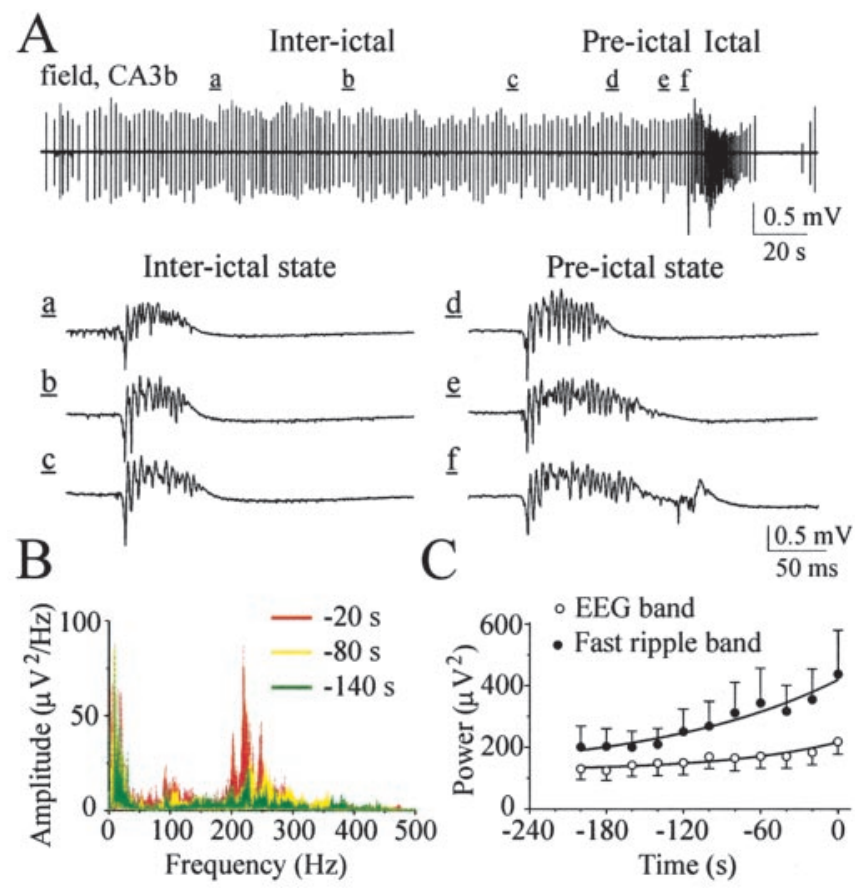

Figure 4. Changes in IED power spectra before transition to ictal-like activity. A, Continuous extracellular record of interictal and ictal-like activity in the CA3b pyramidal cell layer. The trace is selected from simultaneous extracellular field recordings in the CA1, CA3a, CA3b, and CA3c regions of P16 rat hippocampal slice. Epileptiform activity was induced by high $\mathrm{K}^{+}(8.5 \mathrm{~mm})$ bath application. $A, a-f$, Examples of IEDs during interictal state and before transition to ictallike activity. IED duration progressively increased in secondary afterdischarges appeared during the 20-30 sec period before ictal-like activity generation. $B$, Power spectra of interictal epileptiform discharges in $20 \mathrm{sec}$ windows preceding transition to ictal-like activity. C, Averaged IED power in frequency bands from 1 to $100 \mathrm{~Hz}$ (EEG band) and from 200 to $500 \mathrm{~Hz}$ (fast field ripple band) in each $20 \mathrm{sec}$ window preceding transition to ictal-like activity. Data from 10 extracellular field potential recordings in the CA3a and CA3b pyramidal cell layer in five P10-P16 rat hippocampal slices.

tion progressively increased because secondary afterdischarges appeared during the 25-30 sec period before ictal-like activity generation. These secondary afterdischarges reinforced the hippocampal CA3 network excitability, reverberating between the CA3a and CA3c subregions with intervals of 50-100 msec (Fig. 6) and setting the pace of ictal-like-sustained high-frequency oscillations. Ictal-like-sustained discharges were initiated in the CA3c subregion and propagated to CA3a and CA1 (Figs. 5, 6). Ictal-like intermittent discharges were initiated either in CA3c or CA3 a-b.

Similar results were obtained by studying the generation and propagation of 4-AP-induced epileptiform activity (Chesnut and Swann, 1988). In the hippocampal slices prepared from P10-P14 rats, continuous bath application of 4-AP $(100 \mu \mathrm{M})$ elicited IEDs that originated in area CA3 and propagated to CA1 $(n=8)$. Averaged burst length in CA3a was $212 \pm 25 \mathrm{msec}$, and the interburst interval was $3.3 \pm 0.5 \mathrm{sec}$. In $60 \mathrm{sec}$ periods before ictallike activity generation ( $75 \% ; n=6$ of 8 slices), burst length changed to $263 \pm 28 \mathrm{msec}(n=6 ; p=0.2)$, and interval between bursts increased to $4.4 \pm 0.5 \mathrm{msec}(n=6 ; p=0.11)$. Secondary afterdischarges of $0.1-1 \mathrm{sec}$ duration appeared $38 \pm 10$ sec before prolonged ictal-like activity and were not included in burst length. The averaged time delay between IEDs that originated in CA3 $\mathrm{a}$ and propagated to CA3c areas decreased from $17.7 \pm 1.2$ $\operatorname{msec}$ to $10.3 \pm 0.7 \mathrm{msec}(n=4 ; p=0.002$; variance, 6 and 2.1$)$. In pre-ictal states, $52 \pm 7 \%$ of IEDs originated in the CA3c-hilar region, and reverberations of secondary afterdischarges from CA3a to CA3c enhanced the output of the CA3c network (Fig. $5 B$ ). 


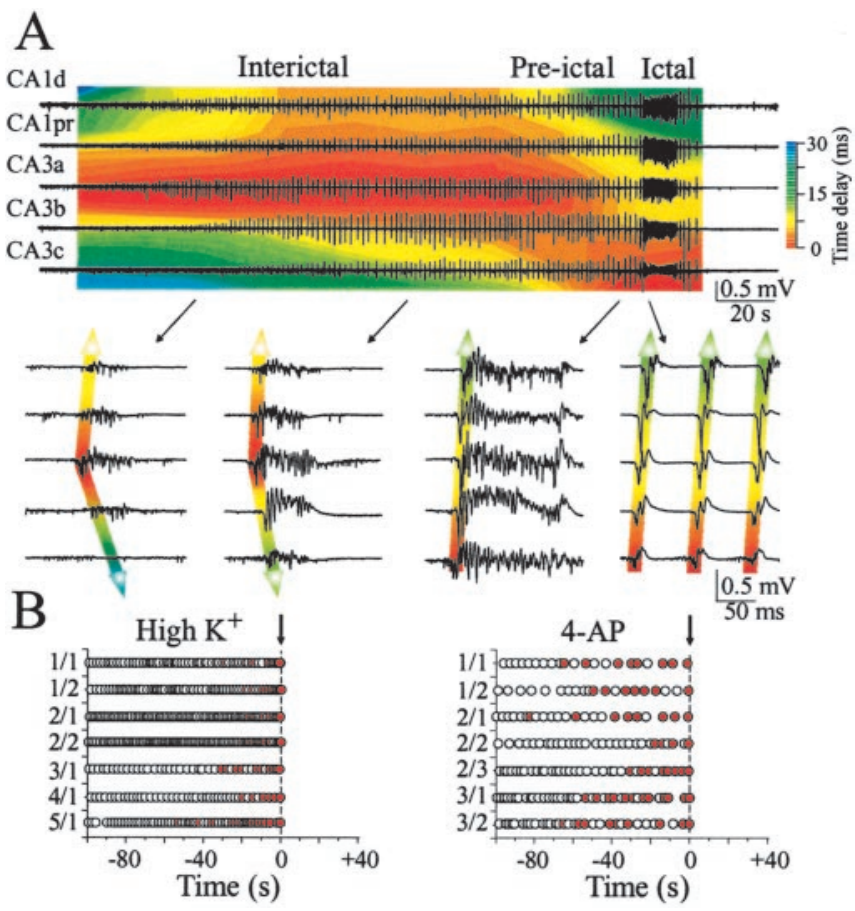

Figure 5. Pre-ictal alterations in hippocampal network activity. $A$, Extracellular field recordings from five electrodes placed in CA3a, CA3b, CA3C, and CA1 (proximal and distal to CA3a) pyramidal cell layers in P16 hippocampal slice preparation (interelectrode distances, $0.6 \mathrm{~mm}$ ). The relative time delays of all IEDs and ictal epileptiform discharges are shown as pseudocolors. Colors were derived using a sliding $1 \mathrm{msec}$ time window, with onsets measured from the (negative) peak of the initial population spike. The IED initiation area shifts before ictal-like epileptiform activity from CA3a to CA3c-hilus, and ictal-sustained discharges originate in CA3c. Bottom traces show the origin and propagation of epileptiform discharges on an extended time scale. $B$, Ictal activity onset can be anticipated on the basis of the shift in the area of origin of pre-ictal IEDs. Open circles correspond to the IEDs initiated in CA3a and propagated to CA3C; colored circles indicate reversed IED propagation (i.e., IEDs originating in CA3c and propagating to CA3a). Several examples are shown for seizures produced by high extracellular $\mathrm{K}^{+}$(left) and the $\mathrm{K}^{+}$-channel blocker 4-AP (100 $\mu \mathrm{m}$; right). For each example, the slice number as well as the seizure number for that slice (slice/seizure number) are indicated along the origin of each panel.

\section{CA3 network paces ictal epileptiform activity}

In the next experiments, we tested the hypothesis that the isolated CA3 network can drive both the ictal-like epileptiform discharges and IEDs. We compared the frequency and gradients of propagation of epileptiform discharges induced by $8.5 \mathrm{~mm}$ extracellular potassium in the isolated portions of hippocampus (Fig. 7). Cutting the Schaffer collaterals in the CA2 area in addition to dissection of perforant path input did not affect ictal-like activity in the CA3 portion but completely abolished both ictal and interictal epileptiform patterns in CA1 $(n=8)$. Extracellular records from the pyramidal cell layer in the CA1 area, separated from Schaffer collaterals and entorhinal cortex, revealed only noncoherent high-frequency MUA (Fig. 7A). IEDs in the CA3 portion were propagated from the $\mathrm{CA} 3 \mathrm{a}-\mathrm{b}$ to $\mathrm{CA} 3 \mathrm{c}$, and their propagation was altered pre-ictally. Ictal-like sustained discharges were initiated in CA3c and back propagated to CA3a.

Sectioning the CA3 area of the hippocampus did not reverse the $\mathrm{CA} 3 \mathrm{a}-\mathrm{CA} 3 \mathrm{~b}$ vector of population burst propagation, and the frequency of CA3a bursts was similar to before dissection ( $43 \pm 4$ bursts $/ \mathrm{min}$ and $48.6 \pm 5$ bursts/min, correspondingly; $n=6 ; p=$ 0.39). The dissected CA3c network also paced the IEDs that caused response in the granular cell layer (Fig. $7 B$ ). The frequency of dissected CA3c bursts was significantly lower $(31.6 \pm 2.4$ bursts/min; $n=6 ; p=0.0002$ ) than the frequency of CA3a
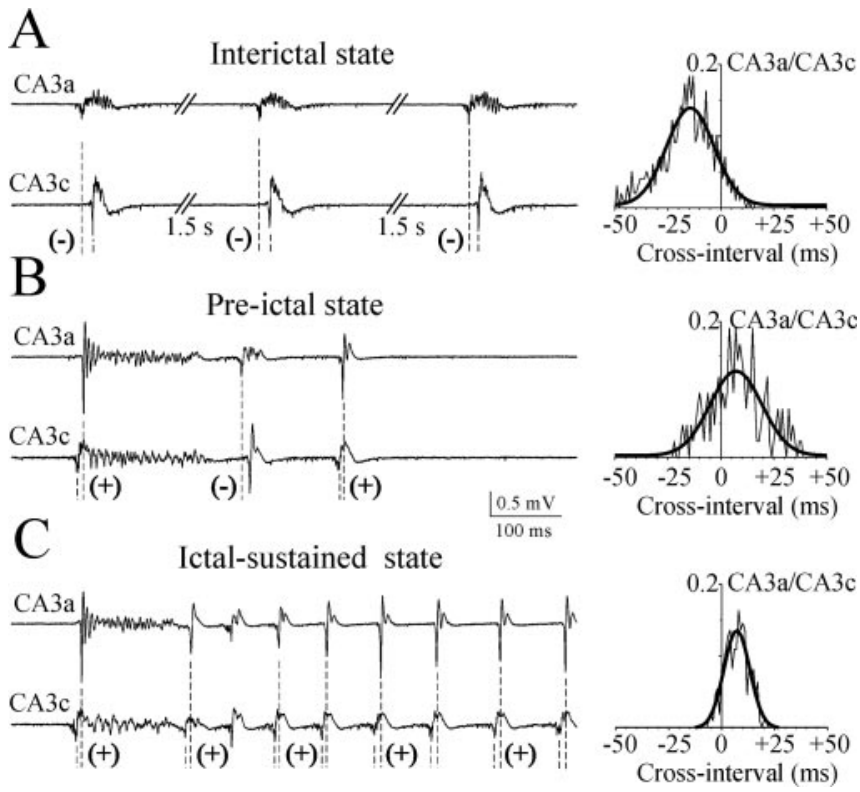

Figure 6. Pre-ictal shift in the IED initiation site and reverberation of secondary afterdischarges. Examples of simultaneous extracellular records from the CA3a and CA3c pyramidal cell layer represent consecutive interictal epileptiform discharges from the steady-state interictal $(A)$, primary IED and secondary afterdischarges from the pre-ictal $(B)$, and ictal-sustained discharges from the ictal ( $C$ ) states. Note also the reverberations of the secondary afterdischarges from CA3a to CA3c in the pre-ictal phase. Population spike and secondary afterdischarge timing are marked with vertical dotted lines; $(+)$ indicates their origin in the CA3c subregion, and $(-)$ indicates origin in CA3a. Cross-correlation histograms represent relative delays in primary population spike and secondary afterdischarge timing in the CA3a area versus CA3c (bin $1 \mathrm{msec}$ ).

bursts, providing evidence for a distributed pacemaker mechanism.

\section{Discussion}

These data indicate that interictal and ictal activity can be initiated within the same neuronal network, and that predictable alterations in interictal activity precede the transition to ictal activity in this network. These findings provide a scientific basis for the long-standing clinical practice of using interictal activity to assess the likelihood of epilepsy and raise new possibilities for seizure prediction strategies.

\section{Previous observations on entorhinal and hippocampal ictal-like activity}

Previous work suggested that reverberation loop activity in the hippocampal-entorhinal circuits represents the central event in the functional organization of hippocampal epileptic discharges (Pare et al., 1992; Stringer and Lothman, 1992). In some in vitro models, the ictal-like epileptiform discharges originated in the entorhinal cortex and propagated via the dentate dyrus to the hippocampus proper, from where they may re-enter the entorhinal cortex (Rafiq et al., 1993; Barbarosie and Avoli, 1997; Barbarosie et al., 2000). Investigations in in vivo animal models suggested that sustained rhythm of the epileptic afterdischarges can independently arise in the CA3-CA1 circuitries, and that reverberation of excitation in the hippocampal-entorhinal loop is not critical for the maintenance of epileptic afterdischarges (Bragin et al., 1997), and that hypersynchronous ictal events originated predominantly in hippocampus (Bragin et al., 1999b). In agreement with in vivo models, our data from multiple-site recordings and lesion experiments in juvenile rat brain slices revealed that onset and maintenance of hippocampal ictal-like activity is indepen- 


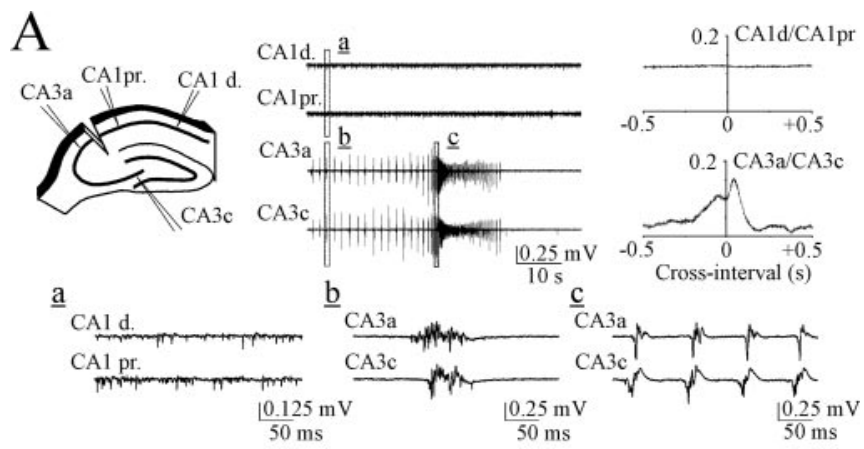

B

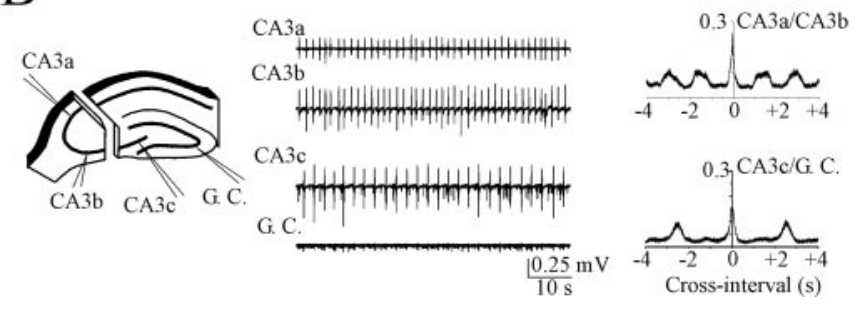

Figure 7. CA3 network paces interictal and ictal-like epileptiform activity. $A$, Severing the connections between $C A 3$ and $C A 1$ areas abolished interictal and ictal-like epileptiform activity in the CA1 region but did not affect epileptiform activity in CA3. Right, Cross-correlations of the MUA detected in the distal CA1 area versus proximal CA1 (top) and in the CA3a versus CA3C (bottom). Two peaks correspond to the interictal phase discharges initiated by CA3a (left peak) and pre-ictal phase discharges initiated by $\mathrm{CA3C}$ (right peak). The events marked by small letters $(a-c)$ are shown on an extended time scale. $B$, Scheme of extracellular field recordings in the isolated portions of hippocampus. Electrodes are positioned in the pyramidal cell layer of the isolated CA3a-CA3b and CA3c dentate gyrus subfields. Right, Cross-correlations of the MUA in CA3a versus $C A 3 b$ (top) and in CA3 c versus granular cell layer of the dentate gyrus (bottom). The frequency of the $C A 3 a-b$ generator was higher than the frequency of the $C A 3 c$ generator. $A, B$, Epileptiform discharges induced by $8.5 \mathrm{~mm}\left[\mathrm{~K}^{+}\right]_{0}$.

dent of entorhinal cortex circuitries (Fig. 3). Nonlinear interaction between IEDs and secondary afterdischarges reverberating between the CA3a and CA3c subregions reinforce the hippocampal CA3 network output, setting the scene for ictal-sustained high-frequency oscillations. The nonessential role of the entorhinal cortex in the generation of ictal activity and the difference in subregional onset of CA3 interictal activity in these experiments versus previous reports (Korn et al., 1987; Barbarosie and Avoli, 1997) are most likely attributable to age and species differences (Weissinger et al., 2000; Colom and Saggau, 1994). Some of these differences may relate to the strain of rat used, thickness of slice, and other technical issues that affect in vitro experiments.

\section{Hippocampal IED origin and propagation}

The initial site of IEDs was determined by the time of negative peak of the initial population spikes (Fig. 2). The CA3 a-b IED pacemaker region correlated with the area of the highest spontaneous MUA in the CA3 a-b pyramidal cell layer (Fig. 1). Recurrent axon collaterals of CA3 pyramidal cells make extensive excitatory synapses on the dendrites of neighboring pyramidal cells (Li et al., 1994), and when one pyramidal cell fires, this powerfully excites neighbors (MacVicar and Dudek, 1980; Miles and Wong, 1983). This facilitates the rapid synchronization of neuronal firing that underlies hippocampal population bursts. Analysis of spike discharges suggests that increasing extracellular $\mathrm{K}^{+}$increases the discharge frequency of spontaneously active cells as well as recruits new cells to generate the action potentials. Both intrinsic cellular properties and synaptic events contribute to the generation of neuronal discharges (Cohen and Miles, 2000). It is suggested that a steady inward current flowing from the dendritic trees toward the cell somata is responsible for the population bursts (Wadman et al., 1992). Intensively activated dendritic $\mathrm{GABA}_{\mathrm{A}}$ receptors by the fast discharging interneurons may contribute to the depolarization and excitation of the pyramidal cells (Staley et al., 1995).

Initiated in the CA3 a-b region, IEDs bidirectionally propagate to the CA1 area and to the CA3c subregion, causing responses in the granular cell layer of the dentate gyrus (Fig. 2). Morphological and electrophysiological data support such feedforward and feedback mechanisms. Apical dendrites of the CA1 pyramidal cells are innervated predominantly by the CA3a subregion ( $\mathrm{Li}$ et al., 1994), and dissection of the Schaffer collaterals between CA3a and CA1 in the hippocampal slices abolished IEDs in CA1 by preventing their propagation from the CA3 region (Fig. 7). CA3 pyramidal cells also send axon collaterals back to the hilar region, in which they excite mossy cells and hilar interneurons (Scharfman, 1994a,b; Kneisler and Dingledine, 1995) with or without synchronous excitation of granule cells (Ylinen et al., 1995; Scharfman, 1996).

\section{Fast field ripples during hippocampal IEDs}

Electrophysiological studies in the kainate-treated rats have revealed 200-500 Hz oscillations, termed "fast ripples" (Bragin et al., 1999b). These oscillations may uniquely occur in areas that generate spontaneous seizures. Two similar types of highfrequency field oscillations have been recorded from the entorhinal cortex and hippocampus of patients with medial temporal lobe epilepsy (Bragin et al., 1999a). The first type appears to be the human equivalent of normal ripples in the rat. The second, fast ripples in the frequency range of $200-500 \mathrm{~Hz}$, is found in the epileptogenic region and may reflect pathological hypersynchronous population spikes of bursting pyramidal cells.

We found similar patterns of hippocampal neuronal activity during high $\mathrm{K}^{+}$-induced IEDs in hippocampal slices in vitro (Figs. 2, 4). Spectral analysis of the interictal epileptiform activity in each $20 \mathrm{sec}$ window preceding transition to ictal-like activity revealed a prominent increase of IED power in the frequency range of fast field ripple $(200-400 \mathrm{~Hz})$ (Fig. 4). During these discharges, individual CA3 neurons fire a burst of action potentials during a 50-200 msec depolarization (data not shown). Intraburst spike frequency during IEDs is identical for the individual CA3 pyramidal cells and population spike frequency. Synchronous burst onset of the regional pyramidal cells may reflect large-amplitude and high-frequency $(200-500 \mathrm{~Hz})$ population spikes, termed "fast field ripples." Antagonists of glutamatergic synaptic transmission (10 $\mu \mathrm{M}$ 2,3-dioxo-6-nitro-1,2,3,4,tetrahydrobenzo[f] quinoxaline-7-sulfonamide and $50 \mu \mathrm{M}$ D- $(-)$-2-amino-5-phosphopentanoic acid abolished IEDs $(n=$ 6; data not shown), suggesting the primary synaptic mechanisms of fast field ripple generation and propagation.

\section{Interictal and ictal activity arise from the same neural network}

Electroencephalographic IEDs are observed in the setting of an increased probability for spontaneous seizures in human temporal lobe epilepsy (Gloor, 1991; Sundaram et al., 1999) and in corresponding animal models (Leite et al., 1996). In focal-onset epilepsy, IEDs are localized to the epileptic brain region and frequently disappear after resection of the epileptogenic brain tissue or spontaneous resolution of epilepsy (Wennberg et al., 1998; Salanova et al., 1999). Thus, IEDs are associated with an increased risk for spontaneous seizures, and this is the basis for the diag- 
nostic use of interictal EEG recordings. This association suggests that the same networks under different conditions generate IEDs and seizures. Our data now provide experimental support for this clinical assumption.

\section{Pre-ictal reorganization of hippocampal network activity and seizure prediction}

Seizures are so named because their onset has not been predictable. If the same neural networks generate IEDs and seizures, then it should be possible to study the mechanisms of transition from one form of epileptiform activity to the other. In the hippocampal slices exposed to high extracellular potassium (8.5 $\mathrm{mM})$, the transition from IEDs to ictal-like activity was characterized by: (1) increase in IED afterdischarges; (2) increase in the velocity of IED propagation; and (3) shift in the IED initiation area from CA3a to CA3c-hilus (Figs. 5, 6). These results indicate that the pre-ictal network alterations detected by intracellular recordings (Traynelis and Dingledine, 1988; Jensen and Yaari, 1997) and nonlinear time series analyses (Martinerie et al., 1998) may also be manifest in the initiation and propagation of IEDs. Nonlinear analyses indicate that the EEG becomes less complex and the phase correlation increases (Lehnertz et al., 2001). These changes may reflect a homogenization of neural activity because of the exclusive transmission of the output of the seizureinitiating network (i.e., entrainment by the epileptic focus). This shift is evidenced in vitro by the increase in afterdischarges (i.e., the fraction of time that the hippocampus was involved in epileptic activity). Increases in extracellular $\mathrm{K}^{+}$and the corresponding depolarization of principal cells (Pedley et al., 1976; Traynelis and Dingledine, 1988), as well as alterations in other ionic concentrations or inactivation of voltage- and/or ligand-gated conductances, may underlie the increase in the velocity of propagation and the shift in the site of initiation of the IEDs. The present analysis differs from recent seizure detection work in that it focuses solely on IEDs rather than the entire EEG signal (Jerger et al., 2001). For seizure prediction, this may be a disadvantage if IED frequency is low. In contrast, pre-ictal alterations in IEDs can be detected with much greater computational efficiency than is currently possible with nonlinear techniques: by measuring the difference in population spike timing in CA3a and CA3c, seizure onset could be predicted up to $1 \mathrm{~min}$ in advance (Figs. 5, 6). In vivo experiments will be needed to determine whether these changes in IEDs can be used to predict seizures from the scalprecorded EEG (Stafstrom et al., 2000), although the bandwidth of the surface EEG should not be an obstacle.

\section{References}

Barbarosie M, Avoli M (1997) CA3-driven hippocampal-entorhinal loop controls rather than sustains in vitro limbic seizures. J Neurosci 17:9308-9314.

Barbarosie M, Louvel J, Kurcewicz I, Avoli M (2000) CA3-released entorhinal seizures disclose dentate gyrus epileptogenicity and unmask a temporoammonic pathway. J Neurophysiol 83:1115-1124.

Bautista RE, Cobbs MA, Spencer DD, Spencer SS (1999) Prediction of surgical outcome by interictal epileptiform abnormalities during intracranial EEG monitoring in patients with extrahippocampal seizures. Epilepsia 40:880-890.

Bragin A, Csicsvari J, Penttonen M, Buzsaki G (1997) Epileptic afterdischarge in the hippocampal-entorhinal system: current source density and unit studies. Neuroscience 76:1187-1203.

Bragin A, Engel Jr J, Wilson CL, Fried I, Buzsaki G (1999a) High-frequency oscillations in human brain. Hippocampus 9:137-142.

Bragin A, Engel Jr J, Wilson CL, Vizentin E, Mathern GW (1999b) Electrophysiologic analysis of a chronic seizure model after unilateral hippocampal KA injection. Epilepsia 40:1210-1221.
Buckmaster PS, Dudek FE (1997) Network properties of the dentate gyrus in epileptic rats with hilar neuron loss and granule cell axon reorganization. J Neurophysiol 77:2685-2696.

Chesnut TJ, Swann JW (1988) Epileptiform activity induced by 4-aminopyridine in immature hippocampus. Epilepsy Res 2:187-195.

Cohen I, Miles R (2000) Contributions of intrinsic and synaptic activities to the generation of neuronal discharges in in vitro hippocampus. J Physiol (Lond) 524:485-502.

Colom LV, Saggau P (1994) Spontaneous interictal-like activity originates in multiple areas of the CA2-CA3 region of hippocampal slices. J Neurophysiol 71:1574-1585.

Dreier JP, Heinemann U (1990) Late low magnesium-induced epileptiform activity in rat entorhinal cortex slices becomes insensitive to the anticonvulsant valproic acid. Neurosci Lett 119:68-70.

Dzhala VI, Staley KJ (2003) Excitatory actions of endogenously released GABA contribute to initiation of ictal epileptiform activity in the developing hippocampus. J Neurosci 23:1840-1846.

Gloor P (1991) Preoperative electroencephalographic investigation in temporal lobe epilepsy: extracranial and intracranial recordings. Can J Neurol Sci 18:554-558.

Gotman J (1991) Relationships between interictal spiking and seizures: human and experimental evidence. Can J Neurol Sci 18:573-576.

Heinemann U, Zhang CL, Eder C (1993) Entorhinal cortex-hippocampal interactions in normal and epileptic temporal lobe. Hippocampus 3:89-97.

Holmes GL, Ben Ari Y (1998) Seizures in the developing brain: perhaps not so benign after all. Neuron 21:1231-1234.

Jensen MS, Yaari Y (1988) The relationship between interictal and ictal paroxysms in an in vitro model of focal hippocampal epilepsy. Ann Neurol 24:591-598.

Jensen MS, Yaari Y (1997) Role of intrinsic burst firing, potassium accumulation, and electrical coupling in the elevated potassium model of hippocampal epilepsy. J Neurophysiol 77:1224-1233.

Jerger KK, Netoff TI, Francis JT, Sauer T, Pecora L, Weinstein SL, Schiff SJ (2001) Early seizure detection. J Clin Neurophysiol 18:259-268.

Jones RS (1993) Entorhinal-hippocampal connections: a speculative view of their function. Trends Neurosci 16:58-64.

Jones RS, Heinemann U (1988) Synaptic and intrinsic responses of medical entorhinal cortical cells in normal and magnesium-free medium in vitro. J Neurophysiol 59:1476-1496.

Kneisler TB, Dingledine R (1995) Synaptic input from CA3 pyramidal cells to dentate basket cells in rat hippocampus. J Physiol (Lond) 487:125-146.

Korn SJ, Giacchino JL, Chamberlin NL, Dingledine R (1987) Epileptiform burst activity induced by potassium in the hippocampus and its regulation by GABA-mediated inhibition. J Neurophysiol 57:325-340.

Lehnertz K, Andrzejak RG, Arnhold J, Kreuz T, Mormann F, Rieke C, Widman AG, Elger CE (2001) Nonlinear EEG analysis in epilepsy: its possible use for interictal focus localization, seizure anticipation, and prevention. J Clin Neurophysiol 18:209-222.

Leite JP, Babb TL, Pretorius JK, Kuhlman PA, Yeoman KM, Mathern GW (1996) Neuron loss, mossy fiber sprouting, and interictal spikes after intrahippocampal kainate in developing rats. Epilepsy Res 26:219-231.

Li XG, Somogyi P, Ylinen A, Buzsaki G (1994) The hippocampal CA3 network: an in vivo intracellular labeling study. J Comp Neurol 339:181-208.

Liu Z, Nagao T, Desjardins GC, Gloor P, Avoli M (1994) Quantitative evaluation of neuronal loss in the dorsal hippocampus in rats with long-term pilocarpine seizures. Epilepsy Res 17:237-247.

Lorente de No R (1934) Studies of the structure of the cerebral cortex: II. Continuation of the study of the ammonic system. J Psychol Neurol 46:113-177.

Lothman EW (1994) Seizure circuits in the hippocampus and associated structures. Hippocampus 4:286-290.

Luhmann HJ, Dzhala VI, Ben Ari Y (2000) Generation and propagation of 4-AP-induced epileptiform activity in neonatal intact limbic structures in vitro. Eur J Neurosci 12:2757-2768.

MacVicar BA, Dudek FE (1980) Local synaptic circuits in rat hippocampus: interactions between pyramidal cells. Brain Res 184:220-223.

Martinerie J, Adam C, Le Van QM, Baulac M, Clemenceau S, Renault B, Varela FJ (1998) Epileptic seizures can be anticipated by non-linear analysis. Nat Med 4:1173-1176.

McNamara JO (1994) Cellular and molecular basis of epilepsy. J Neurosci 14:3413-3425. 
Miles R, Wong RK (1983) Single neurones can initiate synchronized population discharge in the hippocampus. Nature 306:371-373.

Ojemann GA (1987) Surgical therapy for medically intractable epilepsy. J Neurosurg 66:489-499.

Pare D, deCurtis M, Llinas R (1992) Role of the hippocampal-entorhinal loop in temporal lobe epilepsy: extra- and intracellular study in the isolated guinea pig brain in vitro. J Neurosci 12:1867-1881.

Paxinos G, Watson C (1986) The rat brain in stereotaxic coordinates. San Diego: Academic.

Pedley TA (1980) Interictal epileptiform discharges: discriminating characteristics and clinical correlations. Am J EEG Technol 20:101-119.

Pedley TA, Fisher RS, Futamachi KJ, Prince DA (1976) Regulation of extracellular potassium concentration in epileptogenesis. Fed Proc 35:1254-1259.

Prince DA, Connors BW, Benardo LS (1983) Mechanisms underlying interictal-ictal transitions. Adv Neurol 34:177-187.

Rafiq A, DeLorenzo RJ, Coulter DA (1993) Generation and propagation of epileptiform discharges in a combined entorhinal cortex/hippocampal slice. J Neurophysiol 70:1962-1974.

Salanova V, Markand O, Worth R (1999) Longitudinal follow-up in 145 patients with medically refractory temporal lobe epilepsy treated surgically between 1984 and 1995. Epilepsia 40:1417-1423.

Scharfman HE (1994a) EPSPs of dentate gyrus granule cells during epileptiform bursts of dentate hilar "mossy" cells and area CA3 pyramidal cells in disinhibited rat hippocampal slices. J Neurosci 14:6041-6057.

Scharfman HE (1994b) Evidence from simultaneous intracellular recordings in rat hippocampal slices that area CA3 pyramidal cells innervate dentate hilar mossy cells. J Neurophysiol 72:2167-2180.

Scharfman HE (1996) Conditions required for polysynaptic excitation of dentate granule cells by area CA3 pyramidal cells in rat hippocampal slices. Neuroscience 72:655-658.

Schwartzkroin PA, Prince DA (1977) Penicillin-induced epileptiform activity in the hippocampal in vitro prepatation. Ann Neurol 1:463-469.
Spencer SS, Spencer DD (1994) Entorhinal-hippocampal interactions in medial temporal lobe epilepsy. Epilepsia 35:721-727.

Stafstrom CE, Lynch M, Sutula TP (2000) Consequences of epilepsy in the developing brain: implications for surgical management. Semin Pediatr Neurol 7:147-157.

Staley KJ, Soldo BL, Proctor WR (1995) Ionic mechanisms of neuronal excitation by inhibitory GABAA receptors. Science 269:977-981.

Stringer JL, Lothman EW (1992) Reverberatory seizure discharges in hippocampal-parahippocampal circuits. Exp Neurol 116:198-203.

Sundaram M, Sadler RM, Young GB, Pillay N (1999) EEG in epilepsy: current perspectives. Can J Neurol Sci 26:255-262.

Traynelis SF, Dingledine R (1988) Potassium-induced spontaneous electrographic seizures in the rat hippocampal slice. J Neurophysiol 59:259-276

Wadman WJ, Juta AJ, Kamphuis W, Somjen GG (1992) Current source density of sustained potential shifts associated with electrographic seizures and with spreading depression in rat hippocampus. Brain Res 570:85-91.

Walther H, Lambert JD, Jones RS, Heinemann U, Hamon B (1986) Epileptiform activity in combined slices of the hippocampus, subiculum and entorhinal cortex during perfusion with low magnesium medium. Neurosci Lett 69:156-161.

Weissinger F, Buchheim K, Siegmund H, Heinemann U, Meierkord H (2000) Optical imaging reveals characteristic seizure onsets, spread patterns, and propagation velocities in hippocampal-entorhinal cortex slices of juvenile rats. Neurobiol Dis 7:286-298.

Wennberg R, Quesney F, Olivier A, Rasmussen T (1998) Electrocorticography and outcome in frontal lobe epilepsy. Electroencephalogr Clin Neurophysiol 106:357-368.

Wieser HG, Engel Jr J, Williamson PD, Babb TL, Gloor P (1993) Surgically remediable temporal lobe syndromes In: Surgical treatment of the epilepsies (Engel Jr J, ed), pp 49-63. New York: Raven.

Ylinen A, Soltesz I, Bragin A, Penttonen M, Sik A, Buzsaki G (1995) Intracellular correlates of hippocampal theta rhythm in identified pyramidal cells, granule cells, and basket cells. Hippocampus 5:78-90. 